\title{
Advanced imaging in acute stroke management-Part II: Magnetic resonance imaging
}

\author{
Monica Saini, Ken Butcher \\ Department of Medicine, Stroke Unit, Division of Neurology, University of Alberta, Canada
}

Address for correspondence:

Dr. Ken Butcher,

Stroke Unit, Division of Neurology,

University of Alberta, Canada.

E-mail: ken.butcher@ualberta.ca

PMID: 19934552

DOI: $10.4103 / 0028-3886.57792$

\begin{abstract}
Worldwide, non-contrast computed tomography (NCCT) has been the imaging modality of choice in acute stroke, primarily due to its accessibility and short acQuisition time. However, magnetic resonance imaging (MRI), specifically diffusion-weighted imaging (DWI), is much more sensitive to hyperacute ischemic changes. Multimodal MRI studies provide significantly more pathophysiological and prognostic information than NCCT. Finally, perfusion-weighted MRI permits visualization of the extent of the ischemic penumbra in individual patients. MRI-based penumbral imaging appears to be the most promising approach to expanding the thrombolysis population base to include patients with prolonged symptoms. This article describes advances in MRI techniques and their application to acute stroke management.
\end{abstract}

Key words: Diffusion-weighted imaging, hemorrhagic stroke, ischemic stroke, magnetic resonance imaging, penumbra, perfusion-weighted imaging.

\section{Introduction}

In patients with stroke, rapid diagnosis is essential for appropriate management. Results of acute stroke trials amply demonstrated that early treatment is critical in determining prognosis. In the 1980s, magnetic resonance imaging (MRI) scanners were first made available and generated great excitement owing to early recognition of ischemic brain tissue. ${ }^{[1]}$ Advances in MRI techniques have now made it possible to differentiate areas of preventable infarction and identify patients most likely to benefit from interventional therapy.

Conventional spin echo (SE) and gradient recall echo (GRE) images rely on multiple radiofrequency (RF) excitations to generate images. Echoplanar Imaging (EPI) allows sampling of an entire volume of tissue with a single RF pulse excitation. This is accomplished through high-performance gradients, which are cycled on and off during a single period of proton relaxation.
This ultra-fast technique therefore allows imaging of physiological phenomena, including water diffusion and blood flow. Images of the whole brain can be acquired within one minute. ${ }^{[2]}$ The EPI technique can be applied to conventional SE or GRE images.

\section{Imaging the Parenchyma}

\section{Diffusion-weighted imaging}

Diffusion-weighted imaging (DWI) delineates regions of bio-energetic compromise, where sodiumpotassium ATPase activity has failed. DWI employs serial de-phasing and re-phasing gradients, known as diffusion-gradients, to image the movement of protons (water) in space (diffusion). Protons that are not moving (restricted diffusion/cytotoxic edema) are associated with higher signal, i.e. hyper-intensity, on DWI. Under ischemic conditions, DWI restriction occurs within minutes and persists for 7-10 days, at which point cell membranes break down and water is no longer compartmentalized. Vasogenic edema appears hyperintense on T2 images, which will also 
"shine-through" on DWI sequences acquired in subacute stroke patients.

The apparent diffusion coefficient (ADC) is derived from the raw diffusion-weighted images. It is a quantitative measure of the rate of proton, and therefore water molecule, movement. ${ }^{[3]}$ By comparing signal intensities at varying diffusion gradient strengths, the speed of water diffusion can be estimated. Gradients are applied in a number of directions (generally at least three). The strength of gradients is denoted by the symbol ' $b$ '; clinical DWI is normally obtained with a b-value of 1000 . The signal is averaged to form a direction-independent (isotropic) image (sometimes referred to as the 'trace' image). When diffusion gradients are not applied $(b=0)$, a T2-weighted image is obtained. Color-coded or intensity-coded ADC maps are obtained by applying an equation (Stejskal-Tanner) to the signal intensities with two or more b-values for each voxel. ${ }^{[4]}$ In areas of more profound energetic compromise and sequestration of water molecules ADC values are lower. ADC values begin to increase 5-10 days after stroke symptom onset, which can result in the phenomenon of ADC 'pseudoreversal'. In areas of encephalomalacia and vasogenic edema, ADC values are increased, reflecting more freely diffusing water. ${ }^{[5]}$

\section{Perfusion-weighted imaging}

Perfusion-weighted images are currently acquired using the dynamic-susceptibility contrast imaging technique. This is very similar to the computed tomography perfusion (CT perfusion) technique described in Part I of this series. A series of susceptibility-weighted images (known as T2*) are obtained every 1-2 sec during an injection of intravenous gadolinium contrast. As contrast transits the cerebral circulation, MRI T2* signal intensity of the images successively decreases due to the paramagnetic nature of the contrast, and then returns to normal. This change in signal intensity is plotted as a function of time (the signal-intensity time curve). Just as in CTP imaging, the central volume principle is used to calculate cerebral blood flow (CBF) and cerebral blood volume (CBV) on a voxel-wise basis. CBF is proportional to the amplitude of the signal intensity time curve, while CBV is estimated from the area under the signal-intensity time curve. ${ }^{[6]}$ Areas of hypoperfusion can also be visualized as tissue with delayed time to peak (TTP) or prolonged mean transit time (MTT). Although not always performed in real time, most PWI studies have also used the deconvolution technique to correct for delay and dispersion of contrast bolus prior to arrival in the brain. ${ }^{[7,8]} \mathrm{A}$ commonly used parameter in these studies is Tmax, which is simply the time to peak after deconvolution of the signal-intensity time curve. ${ }^{[9]}$ Voxel-wise values for each parameter are assigned a color code or intensity value, and maps are then constructed for CBF, CBV, TTP, Tmax and MTT.

In the near future, it may be possible to routinely measure cerebral perfusion without the need for gadolinium injections. Arterial spin labeling (ASL) is a quantitative MR perfusion method that does not require paramagnetic contrast. ${ }^{[10]}$ An inversion pulse is utilized to tag protons in the blood prior to their entry into the imaging plane. In this way, water flowing in blood is used as an endogenous perfusion tracer. CBF maps are generated by subtraction of control from the spin-labeled images. The signal to noise ratio is relatively low, and significant contamination from blood-oxygenation-level-dependent effects is seen. ${ }^{[11]}$ As no gadolinium is used, ASL can be used for sequential study of $\mathrm{CBF}$ without fear of contrastrelated adverse effects. However, this technique is not routinely performed and is still a research tool. In most clinical settings, bolus contrast PWI methodology is currently used to visualize perfusion deficits.

\section{Imaging Blood Vessels: Magnetic Resonance Angiography}

Magnetic resonance angiography (MRA) can be performed using the time of flight (TOF) or the phase contrast technique. In TOF studies, repetitive pulses are used to saturate tissue, while mobile protons in the vessels create a signal that is utilized to create images of the vasculature. Areas with turbulent flow, slow flow and adjacent fat or blood products can lead to erroneous estimation of vessel lumen diameter and patency. ${ }^{[12]}$ Depending on the sequence parameters, the acquisition time for a 3D TOF is approximately 3-6 $\mathrm{min} \cdot{ }^{[13]}$ The phase contrast technique is based on the manipulation of phase of magnetization, and is sensitive to flow velocities. ${ }^{[14]}$ In contrast to TOF, phase contrast MRA can therefore indicate both the rate and direction of flow, although signal-to-noise ratios are lower and this technique is less often used in clinical practice.

Contrast-enhanced (CE) MRA utilizes gadolinium as an intravascular contrast medium. CE-MRA has a higher signal-to-noise ratio compared to conventional MRA, and visualization of smaller intracranial vessels (beyond the proximal middle cerebral artery, MCA) is improved. ${ }^{[15]}$ Conventional 3D TOF tends to overestimate stenotic lesions while CE-MRA provides a better morphological image of the vessels. ${ }^{[16]}$ MRA has a satisfactory sensitivity and specificity $(80-90 \%)$, as compared to intra-arterial angiography, for detection of high-grade stenosis or occlusion of the internal carotid (ICA). For moderately severe ICA stenosis and for assessment of intracranial vessels, the sensitivity is less than optimal $(0-18 \%$ for intracranial stenosis $\geq 50 \%)$. ${ }^{[17,18]}$ 


\section{Imaging the Ischemic Penumbra: PWI-DWI Mismatch}

As discussed in the first article, the extent of the ischemic penumbra is fundamental to the success of all stroke therapies, including thrombolysis. Therefore demonstration of the penumbra, or lack thereof, in all stroke patients prior to making therapeutic decisions is ideal. Although a model of the penumbra based solely on perfusion thresholds is possible [Figure 1], combining DWI and PWI allows a visual assessment of the pathophysiological changes occurring in ischemic stroke. The DWI lesion generally represents the ischemic core [Figures 1 and 2], although it is now recognized that a portion of this tissue is sometimes part of the penumbra. This is because tissue with moderately decreased ADC, generally less than $15 \%$ of normal, sometimes escapes infarction. ${ }^{[19,20]}$ This phenomenon is known as DWI reversal. ${ }^{[21]}$ Nonetheless, mismatch between a larger PWI abnormality and a smaller DWI lesion has been postulated to represent the ischemic penumbra ${ }^{[22]}$ Within the first $3-6 \mathrm{~h}$ after ischemic stroke onset, up to $80 \%$ patients have PWIDWI mismatch, but the frequency of penumbral patterns decreases progressively with duration of symptoms. ${ }^{[23,24]}$

One of the technical difficulties that has limited immediate use of mismatch models in routine clinical practice is the lack of a standardized and objective definition. ${ }^{[25]}$ It is impossible to define perfusion thresholds for infarct core/penumbra, as they vary with duration of ischemia and can therefore be modified by recanalization and/or collateral circulation. ${ }^{[26]}$ As described above, there are a number of perfusion parameters, i.e. TTP, MTT, CBF that can be used to define the area at risk. ${ }^{[27]}$ Finally, it is not clear what constitutes a critical volume of mismatch tissue. A value of $20 \%$, i.e. perfusion deficits $\geq 120 \%$ of DWI lesion volume, has been utilized in a number of studies, but this is an arbitrary ratio. Comparative studies with PET do confirm that visual inspection of TTP maps, without objective thresholds does result in an over-estimation of the true volume at risk, due to the inclusion of areas of benign oligemia. ${ }^{[28,29]}$

\section{Evidence for the mismatch hypothesis with respect to MRI-based selection of patients for thrombolysis}

Patients with a PWI-DWI mismatch pattern have been hypothesized to be more likely to benefit from reperfusion

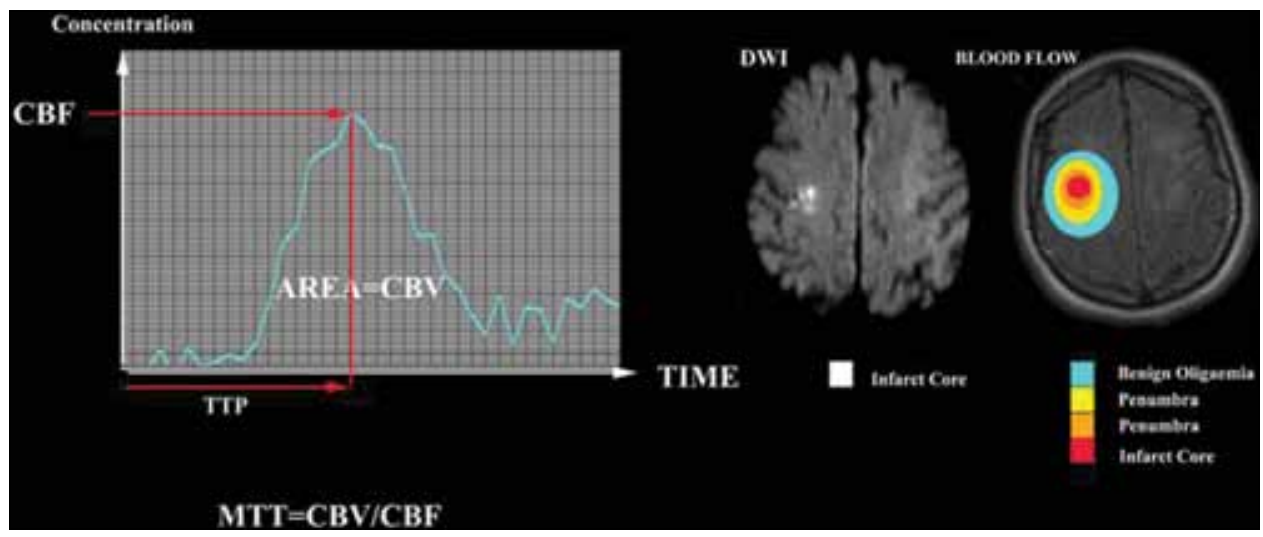

Figure 1: Signal-intensity time curve used to generate perfusion-weighted images. Peak intensity is proportional to cerebral blood flow, area under the curve to cerebral blood volume. Mean transit time is calculated as CBV/CBF. Diffusion-weighted image demonstrates the infarct core. The volume of tissue that is hypoperfused, but outside the infarct core can be used as an operational definition of the ischemic penumbra. Penumbral tissue volume estimates vary with the PWI parameter utilized and the threshold used to differentiate at risk tissue from benign oligemia

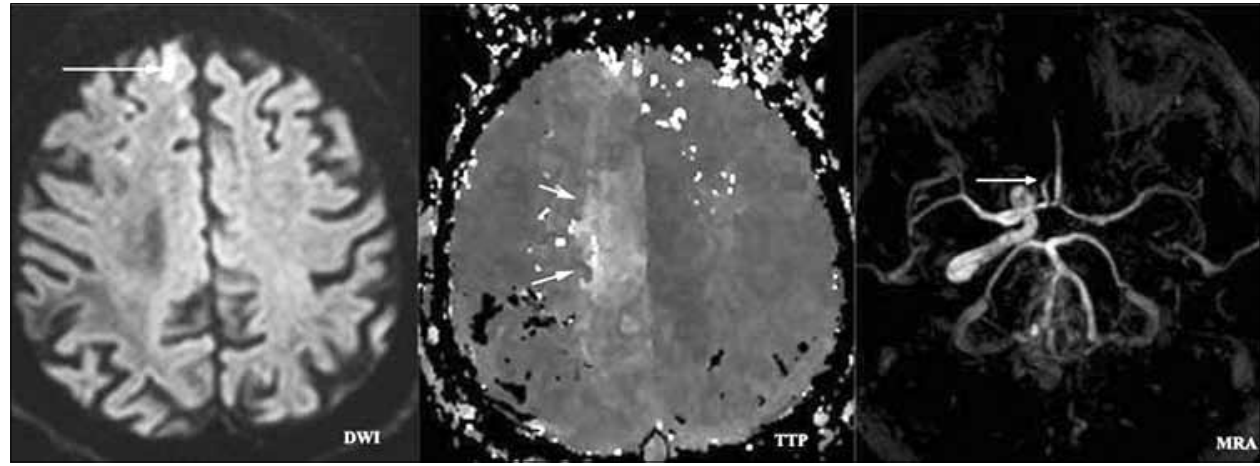

Figure 2: Acute right anterior cerebral artery stroke. The diffusion-weighted image shows patchy areas of restricted diffusion (infarct core).

Perfusion-weighted imaging, specifically a time to peak map, demonstrates hypoperfusion in the ACA territory. A significant PWI-DWI mismatch is evident. Magnetic resonance angiography shows right A2 occlusion (arrow). In addition, the left internal carotid artery is not visualized due to a chronic occlusion 
therapy relative to those without a mismatch. ${ }^{[30]}$ In a cohort of 24 patients, imaged within $6 \mathrm{~h}$ of stroke onset, Schellinger et al., administered open label tissue plasminogen activator (tPA) to all patients with visible PWI-DWI mismatch. ${ }^{[3]}$ Patients who recanalized after receiving tPA had better clinical outcomes. Although this study did not confirm the mismatch hypothesis, it did demonstrate that PWI-DWI selection of acute thrombolysis patients is feasible. Similarly, Parsons et al., also evaluated the effect of PWI-DWI mismatch in patients thrombolysed within $6 \mathrm{~h}$ of stroke onset. ${ }^{[32]}$ In this study, all patients were treated with tPA, regardless of the MRI findings. Mismatch patients who received tPA had higher rates of recanalization and reperfusion, relative to non-mismatch patients, which was associated with significant increases in the volume of penumbral tissue that was salvaged. ${ }^{[32]}$

Several recent clinical studies of intravenous thrombolysis have included pre-treatment MRI PWI-DWI. ${ }^{[33-36]}$ The diffusion and perfusion imaging evaluation for understanding stroke evolution (DEFUSE) study tested the hypothesis that the benefit of early reperfusion, with thrombolysis, would be enhanced in patients with mismatch. ${ }^{[35]}$ Intravenous tPA was administered to all patients with ischemic stroke (3-6 h) regardless of their MRI profiles. Tmax maps, with $\geq 2$ sec delay, were used to calculate the perfusion lesion volumes and reperfusion was defined as a $30 \%$ or more and $10 \mathrm{ml}$ or greater reduction in PWI volumes on follow-up scans. An interim analysis indicated that a number of different PWI-DWI combinations could result in a mismatch by volume, but each had different prognostic significance. Mismatch profiles were divided into "small lesion" (PWI and DWI $<10 \mathrm{ml}$ ), "malignant profile" (baseline DWI lesion $\geq 100 \mathrm{ml}$ and/or PWI lesion $\geq 100 \mathrm{ml}$ with Tmax delay $\geq 8 \mathrm{sec}$ ) and "target mismatch" (PWI $\geq 120 \%$ of DWI; PWI $\geq 10 \mathrm{ml}$ more than DWI, without malignant profile). At baseline, 54\% showed PWI-DWI mismatch, and a small lesion profile was seen in $26 \%$ of the study population. The frequency of favorable clinical outcomes was significantly increased in target mismatch patients with early reperfusion, relative to those with persisting PWI deficits. Patients with the malignant profile had a low rate of favorable outcome, and $100 \%$ symptomatic intracranial hemorrhage rate if reperfusion occurred. In contrast, all patients with a small lesion profile had favorable outcomes. These results suggested that the mismatch hypothesis requires further refinement in order to identify a subgroup of patients (target mismatch) most likely to derive benefit from use of thrombolytic agents. ${ }^{[35]}$

The Echoplanar Imaging Thrombolytic Evaluation Trial (EPITHET) was designed specifically to test the hypothesis that tPA would result in attenuation of infarct expansion in patients with PWI-DWI mismatch. ${ }^{[36]}$ As in DEFUSE, patients with ischemic stroke $(n=100) 3-6 \mathrm{~h}$ after symptom onset were enrolled without reference to the baseline MRI results. Unlike DEFUSE, patients were randomized to tPA or placebo in double-blind design. The a priori definition of mismatch was PWI/DWI ratio $>1.2$ and PWI-DWI volume $\geq 10 \mathrm{ml}$. Using this definition, $86 \%$ of randomized patients had mismatch at baseline. Infarct growth at 90 days, the primary endpoint of the trial, was not significantly different between tPA and placebo-treated patients. There were, however, strong trends to attenuation of DWI lesion expansion in patients treated with tPA. In addition, complete lack of DWI lesion expansion occurred in $23 \%$ fewer patients treated with tPA, relative to placebo. ${ }^{[36]}$ Post-hoc analyses indicate that the mismatch definition used in the primary EPITHET analysis was too liberal and the trial was also underpowered. ${ }^{[37]}$

The Desmoteplase in Acute Ischemic Stroke (DIAS) and the related DEDAS (Dose Escalation of Desmoteplase for Acute Ischemic Stroke) trials were the initial randomized, placebo-controlled trials of a thrombolytic agent that used PWI-DWI mismatch for patient selection ${ }^{[33,34]}$ DIAS patients with $\geq 20 \%$ PWI-DWI mismatch, estimated visually, were eligible to be randomized. Investigators were free to use any PWI parameter they chose to assess mismatch and no thresholds were applied. In most cases, mismatch would therefore have been assessed on the basis of raw TTP maps. A dose-dependent rate of favorable clinical outcome was seen with desmoteplase, as compared to placebo. A post-hoc analysis indicated that reperfusion, defined as either $\geq 30 \%$ reduction of MTT volume of abnormality or $\geq 2$ points improvement on TIMI (adapted Thrombolysis In Myocardial Infarction grading scheme using MRA), was a favorable prognostic factor for clinical outcomes. Intriguingly, no effect of longer stroke onset-to-treatment time was observed on treatment effect in selected patients. This challenged the widely held concept of a "time-window" and suggested that the presence of mismatch, rather than time elapsed since symptom onset, was an important predictor of response to reperfusion therapy. ${ }^{[34]}$ DEDAS, evaluated the safety and efficacy of two-dose tiers of desmoteplase. ${ }^{[33]}$ Patients with $\geq 20 \%$ PWI-DWI mismatch, with a PWI deficit (with or without DWI lesion) of $\geq 2 \mathrm{~cm}$ diameter involving the hemispheric grey matter, were eligible for thrombolytic therapy. In patients fulfilling all MRI criteria, the use of desmoteplase $(125 \mu \mathrm{g} / \mathrm{kg})$ was associated with favorable clinical outcomes. Low rates of symptomatic intracranial hemorrhage (sICH) were observed in selected patients, indicating that beyond the $3 \mathrm{~h}$ window, PWIDWI MRI was potentially useful in identifying patients most likely to benefit from reperfusion.

Although studies to date support the concept of 
penumbral selection for thrombolysis in an extended therapeutic window, it is also evident that the hypothesis requires further refinement. What is clear is that more conservative definitions of mismatch are required, based on higher PWI thresholds and/or ratios greater than $20 \%$. Future trials will utilize these more conservative definitions in patient inclusion criteria. We are optimistic that penumbral assessment tools will ultimately improve thrombolysis patient selection.

\section{Alternative Resonance Imaging-Based Penumbral Selection Paradigms}

Imaging blood flow with either MRI or CT is not routine in many stroke treatment centers. In addition, the EPITHET and DEFUSE studies have made it clear that more advanced tools than simple TTP maps, without a threshold applied, are required to accurately define significant hypoperfusion. ${ }^{[35,36]}$ An alternative definition has therefore been devised that utilizes the severity of the clinical deficits as a surrogate marker for blood flow measurement. The "clinical-diffusion" mismatch (CDM), originally described by Davalos et al., defined CDM as an National Institute of Health Stroke Scale NIHSS $\geq 8$ associated with DWI lesion volume $\leq 25 \mathrm{~mL}$. In a cohort of 166 patients with hemispheric stroke of $<12 \mathrm{~h}$ duration, CDM was detected in 52\%. CDM was found to be a predictor of lesion growth and early neurological deterioration (increase in NIHSS $\geq 4$ at $72 \mathrm{~h}$ ). ${ }^{[38]}$ Prosser et al., evaluated CDM in 54 patients imaged within $6 \mathrm{~h}$ of stroke onset and compared it to PWI-DWI mismatch. CDM detected PWI-DWI mismatch with high specificity (93\%) but lower sensitivity (53\%). Furthermore, DWI lesion expansion was significantly greater in patients with CDM as compared to those without CDM.[39] Thus CDM may be a useful surrogate for penumbral tissue patterns, although the additional information provided by blood flow imaging is fundamental to the pathophysiology of ischemic stroke and therefore should be obtained whenever possible, provided this does not result in undue delays to acute therapy.

Studies such as DEFUSE and EPITHET have all utilized 'off-line' planimetric volume measurements. ${ }^{[35,36]}$ While this is accurate, it is not yet automated and therefore impractical in the setting of acute stroke patient care, where a decision regarding thrombolysis must be made in minutes. An alternative to planimetric measurements is the use of an ordinal scale to rate the severity of DWI and PWI deficits. It has been demonstrated that the ASPECT score can be applied to DWI and PWI images ${ }^{[40,41]}$ Both DWI and PWI ASPECT scores are correlated with planimetric volumes. ${ }^{[41]}$ The 'MR Mismatch score' is a measure of mismatch volume, that can be calculated by subtracting DWI ASPECT from PWI ASPECT scores..$^{[40]}$
MR Mismatch scores predict planimetric mismatch volumes with high specificity.

An MRA-DWI mismatch model has also been proposed on the basis of DWI lesion volume and proximal vessel occlusion identified on MRA [Figure 2]. ${ }^{[42]}$ In the DEFUSE study patients with proximal vessel occlusion and DWI lesion volume $<25 \mathrm{ml}$, and those with proximal vessel stenosis and DWI lesion volume $<15 \mathrm{ml}$ (MRA-DWI mismatch) benefitted from reperfusion therapy administered 3-6 h from symptom onset. ${ }^{[42]}$ Vessel occlusion associated with evidence of limited parenchymal injury is a logical treatment target, although the lack of blood flow imaging may preclude some patients with penumbral patterns secondary to smaller vessel occlusions, not visible on MRA or CTA, from treatment.

\section{Magnetic Resonance Imaging Imaging-Based Prediction of tPA-Related Bleeding Risk}

Baseline DWI lesion volume has been studied as a potential predictor of hemorrhagic transformation following tPA. In some studies larger DWI lesion volumes have been associated with increased hemorrhage rates. In the DEFUSE study also, larger DWI lesion volumes were associated with higher hemorrhage rates. A higher rate of hemorrhage was seen in patients with the "malignant profile", defined as baseline DWI lesion $\geq 100 \mathrm{ml}$ and/ or PWI lesion $\geq 100 \mathrm{ml}$ with Tmax delay $\geq 8 \mathrm{sec}^{\left[{ }^{[35]}\right.} \mathrm{In}$ EPITHET, however, there was no relationship between initial DWI lesion volume and symptomatic hemorrhage, although there were relatively few of these events (four) making statistical test results somewhat less reliable. ${ }^{[36]}$ Although patients with the "malignant profile" had worse clinical outcomes, when treated with tPA, compared to those without the malignant profile, no increase in the risk of sICH was observed, in contrast to results from DEFUSE. ${ }^{[36]}$

A large multicenter study, including 645 patients thrombolysed within $6 \mathrm{~h}$ of stroke onset, retrospectively evaluated the value of DWI lesion volumes (on baseline MRI) in predicting sICH following intravenous or intra-arterial thrombolysis. ${ }^{[43]}$ Patients who developed sICH had significantly larger baseline DWI lesion volumes (44 vs. $18 \mathrm{ml}$ ) compared to those without sICH. Compared to patients with baseline DWI lesion volume $\leq 10 \mathrm{ml}$, the odds of developing sICH were higher in patients with moderate $(10-100 \mathrm{ml}, \mathrm{OR} 2.8)$ and large (>100 ml, OR 5.8) DWI volumes. ${ }^{[43]}$ In contrast to the above reports, Thomalla et al., reported no significant correlation between PWI/DWI lesion volumes and the incidence of $\mathrm{PH}$ following thrombolysis within a 6-h window. However, a significant correlation was 
observed between hemorrhagic transformation (HT) and baseline PWI volumes, suggesting that the mechanisms underlying HT and PH may be different. ${ }^{[4]]}$

Other studies have utilized the severity of diffusionrestriction to predict hemorrhagic transformation. Tong et al., showed that in patients imaged within $8 \mathrm{~h}$ of stroke onset, ischemic areas with higher percentage of pixels with severely restricted diffusion $\left(\right.$ ADC $\left.<550 \times 10^{-6} \mathrm{~mm}^{2} / \mathrm{s}\right)$ were at greater risk for developing HT. ${ }^{[45]}$ Selim et al., retrospectively analyzed MRI-DWI data in patients thrombolysed within a $3 \mathrm{~h}$ window. Larger pre-treatment DWI lesion volume and a higher absolute number of voxels with ADC values $<550 \times 10^{-6} \mathrm{~mm}^{2} / \mathrm{s}$ were associated with increased risk of HT. ${ }^{[6]}$ Several factors, including parenchymal changes, reperfusion, hypertension and glycemic control determine the risk of hemorrhage. Therefore, models based solely on imaging are unlikely to reliably predict the risk of HT, both spontaneous and tPA-related.

\section{Advanced Magnetic Resonance Imaging Imaging and Intracerebral Hemorrhage}

A common concern amongst clinicians who do not routinely use or have access to MRI is the ability to detect acute blood. There is a perceived difficulty associated with visualization of acute hemorrhage on MRI scans. On routine spin echo sequences, intravascular blood is visible due to the effects of flow, which disrupt signal acquisition, resulting in the 'flow-voids'. Factors that alter MRI signal intensity in the presence of extravascular blood include increased red cell density, clot matrix formation, decreasing cellular hydration and blood deoxygenation and breakdown. ${ }^{[47]}$ Blood deoxygenation and breakdown products are the most important, due to their susceptibility properties as well as their effects on relaxivity of adjacent water molecules (protons). Paramagnetic blood constituents and metabolic products include deoxyhemoglobin, methemoglobin and hemosiderin. Oxyhemoglobin is diamagnetic and therefore does not result in changes in MRI signal. Acute blood also contains significant amounts of water in the form of plasma, which is heavily T2-weighted. Thus, acute intraparenchymal blood, has a mixed signal on traditional T2-weighted images [Figure 2]. ${ }^{[47,48]}$

In hyper-acute intracerebral hemorrhage (ICH), the susceptibility effects are first observed at the periphery of the hematoma [Figure 3]. ${ }^{[49]}$ This is related to formation of deoxyhemoglobin and magnetic field inhomogeneities at the interface with surrounding normal diamagnetic tissue. This is useful in delineating the boundary of the hematoma, from the rim of peri-hematomal edema, as the latter is always hyperintense on T2-weighted images. It is important to recognize that measurements of hematoma volume made on MRI will tend to overestimate the true volume. ${ }^{[50]}$ Susceptibility-weighted sequences including GRE T2*, which are more sensitive to the paramagnetic effects of deoxyhemoglobin, make acute blood even more obvious on MRI [Figure 2] ${ }^{[47,51-53]}$ MRI signal changes on GRE, T1 and T2-weighted images can be used to estimate the age of intracranial blood. In addition to the increasingly hypointense $\mathrm{T} 2$ signals, seen with progressive deoxygenation, after $72 \mathrm{~h}, \mathrm{~T} 1$ signal intensity increases due to the presence of methemoglobin. After approximately seven days, the $\mathrm{T} 2$ signal intensity will also increase, as red blood cells are lysed. Eventually, both T1 and T2 signals are significantly decreased, as only hemosiderin is left as a remnant of the hematoma. Depending on the size of the hematoma, a Cerebrospinal fluid CSF-filled cavity, which is heavily T2-weighted, may also be evident.

A number of comparative studies have demonstrated that acute ICH can be readily diagnosed and differentiated from ischemia using MRI. ${ }^{[54,55]}$ MRI is markedly superior to $\mathrm{CT}$ for the detection of chronic blood. This includes identification of cerebral microbleeds (CMBs), defined as hypo-intensities, less than $5 \mathrm{~mm}$ in diameter, most easily identified on susceptibility-weighted sequences. ${ }^{[56-59]}$ These foci of MR signal loss represent deposits of hemosiderin within macrophages, resulting

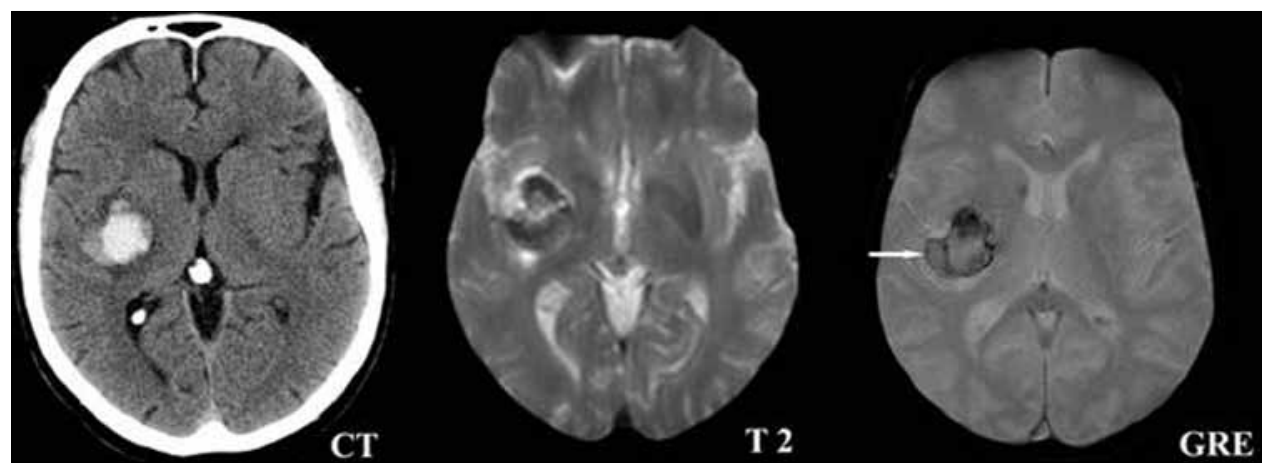

Figure 3: Acute intracerebral (right lentiform nucleus) hemorrhage seen as an area of hyperdensity on non-contrast CT. T2-weighted MRI shows lesion with heterogeneous intensity. Gradient recall echo image shows susceptibility effects (hypointense rim; arrow) at the periphery of the hematoma 
from past bleeding episodes. Histopathological analysis demonstrates a strong association between CMBs and microangiopathy secondary to lipo/fibrohyalanosis and amyloid deposition. ${ }^{[57]}$ The incidence of CMBs in populations without neurological disease has been estimated to be between 4 and $6 \%{ }^{[60,61]}$

It has been proposed that CMBs may be predictors of symptomatic HT following thrombolytic therapy for acute ischemic stroke. There are reports of increased rates of $\mathrm{ICH}$ following both intra-arterial and intravenous therapy in patients with CMBs. ${ }^{[62,63]}$ This association has been particularly compelling in cases where the hemorrhage has appeared to occur at the site of a $\mathrm{CMB}$, sometimes remote from the ischemic region. ${ }^{[63]} \mathrm{A}$ study demonstrated that the presence of CMBs independently predicted HT of ischemic infarcts, irrespective of treatment with tPA. ${ }^{[64]}$ In the BRASIL (Bleeding Risk Analysis in Stroke by T2*-Weighted Imaging before thromboLysis) study, the OR for sICH (following rTPA, given within $6 \mathrm{~h}$ of stroke onset) in patients with CMBs versus patients without $\mathrm{CMBs}$ was 2.23. ${ }^{[65]}$ While CMBs are likely to be associated with increased risk of early hemorrhage after ischemic stroke, it is not clear whether thrombolysis necessarily significantly adds to this risk. The number of CMBs may also have an impact on the risk of hemorrhage ${ }^{[66]}$ However, this has not been adequately evaluated yet. Assessing the absolute risk that CMBs represent to potential thrombolysis candidates will require a very large number of patients, but at this point it does not appear to be excessive.

\section{CT Versus Magnetic Resonance Imaging in Acute Stroke Assessment}

MRI, specifically DWI, is more sensitive than NCCT for detection of early ischemic changes. ${ }^{[40]}$ Using PWI, it is possible to obtain a complete visual representation of the ischemic penumbra. MRI is also the investigation of choice for differentiating stroke from stroke-mimics, which is critical in the emergent setting. ${ }^{[67]}$ In addition, physician concerns about the ability of MRI to detect $\mathrm{ICH}$ or subarachnoid hemorrhage are now known to be unwarranted. MRI actually provides more information about $\mathrm{ICH}$, including etiology in the case of secondary hemorrhage, age of blood products and previous bleeding events. ${ }^{[68-70]}$ Although multimodal MRI provides comprehensive information about perfusion and the extent of ischemia, any additional benefit over multimodal $\mathrm{CT}$ in the first $3 \mathrm{~h}$ following stroke is controversial. ${ }^{[68,71]}$ MRI technology is costly, less accessible hyper-acutely and involves more imaging time than CT. CTA and CTP can also reliably demonstrate vessel occlusion, and salvageable tissue. However, exposure to contrast and a limited coverage of brain tissue remain important concerns with multimodal CT.

It has become increasingly clear that the response to reperfusion therapy is largely determined by the volume of the penumbra. In this context, both multimodal MRI and CT are superior to NCCT for delineation of salvageable tissue and identification of patients who are most likely to benefit from thrombolytic therapy. The choice between multimodal MRI and CT, will vary between centers, depending on availability, time of imaging, and also the characteristics of the patient presenting with stroke symptoms.

\section{Conclusion}

MRI remains the investigation of choice in patients where there is diagnostic uncertainty. It is also one of the most practical means of assessing penumbral tissue in the acute setting. Although advanced imaging in acute stroke does require a significant investment on the part of all involved in the care of these patients, it is worthwhile in our opinion. In the future, simplified and automated assessment tools may facilitate more widespread uptake of these techniques. In the meantime, stroke clinicians would be well advised to understand the concepts underlying MRI acquisition and assessment.

\section{References}

1. Moseley ME, Kucharczyk J, Mintorovitch J, Cohen Y, Kurhanewicz J, Derugin N, et al. Diffusion-weighted MR imaging of acute stroke: correlation with T2 weighted and magnetic susceptibility enhanced MR imaging in cats. A.JNR 1990;11:423-9.

2. Poustchi-Amin M, Mirowitz SA, Brown JJ, McKinstry RJ, Li T. Principles and Applications of Echo-planar Imaging: A Review for the General Radiologist. Radiographics 2001;21:767-79.

3. Desmond PM, Lovell AC, Rawlinson AA, Parsons MW, Barber PA, Yang Q, et al., Tress BM. The value of apparent diffusion coefficient maps in early cerebral ischemia. AJNR Am J Neuroradiol 2001;22:1260-7.

4. Hrabe J, Kaur G, Guilfoyle DN. Principles and limitations of NMR diffusion measurements. J Med Phys 2007;32:34-42.

5. Thulborn KR, Gindin TS, Davis D, Erb P. Comprehensive MRI protocol for stroke management: Tissue sodium concentration as a measure of tissue viability in a non-human primate model and clinical studies. Radiology 1999;139:26-34.

6. Ostergaard L. Principles of cerebral perfusion imaging by bolus tracking. J Mag Res Imag 2005;22:710-7.

7. Calamante F, Gadian DG, Connelly A. Quantification of perfusion using bolus tracking magnetic resonance imaging in stroke: Assumptions, limitations, and potential implications for clinical use. Stroke 2002;33:1146-51.

8. Wu O, Østergaard L, Weisskoff RM, Benner T, Rosen BR, Sorensen AG. Tracer arrival timing-insensitive technique for estimating flow in MR perfusion-weighted imaging using singular value decomposition with a block-circulant deconvolution matrix. Magn Reson Med 2003;50:164-74.

9. Butcher KS, Parsons M, MacGregor L, Barber PA, Chalk J, Bladin C, et al. Refining the perfusion-diffusion mismatch hypothesis. Stroke 2005;36:1153-9.

10. Deibler AR, Pollock JM, Kraft RA, Tan H, Burdette JH, Maldjian JA. Arterial Spin-Labeling in Routine Clinical Practice, Part 1: Technique and Artifacts. AJNR 2008;29:1228-34. 
11. Lu H, Donahue MJ, van Zijl PC. Detrimental effects of BOLD signal in arterial spin labeling fMRI at high field strength. Magn Reson Med 2006;56:546-52.

12. Hilfiker PR, Herfkens RJ, Heiss SG, Alley MT, Fleischmann D, Pelc NJ. Partial fat-saturated contrast enhanced three-dimensional MR angiography compared with nonfat-saturated and conventional fat saturated MR angiography. Radiology 2000;216:298-303.

13. O" zsarlak O", Parizel PM, Van Goethem JW. Low-dose gadolinium enhanced 3D time-of-flight MR angiography of the intracranial vessels using PAT optimized phased array 8-channel head coil. Eur Radiol 2004;14:2067-71.

14. Dumoulin CL, Souza SP, Walker MF, Wagle W. Three-dimensional phase contrast angiography. Magn Reson Med 1989;9:139-49.

15. Marchal G, Michiels J, Bosmans H, Van Hecke P. Contrast enhanced MRA of the brain. J Comput Assist Tomogr 1992;16:25-9.

16. Sohn CH, Sevick RJ, Frayne R. Contrast-enhanced MR angiography of the intracranial circulation. Magn Reson Imaging Clin N Am 2003;11:599-614.

17. Debrey SM, Yu H, Lynch JK, Lövblad KO, Wright VL, Janket SJ, et al. Diagnostic accuracy of magnetic resonance angiography for internal carotid artery disease: A systematic review and meta-analysis. Stroke 2008;39:2237-48.

18. Wright VL, Olan W, Dick B, Yu H, Alberts-Grill N, Latour LL, et al. Assessment of CE-MRA for the rapid detection of supra-aortic vascular disease. Neurology 2005;65:27-32.

19. Desmond PM, Lovell AC, Rawlinson AA, Parsons MW, Barber PA, Yang $Q$, et al. The value of apparent diffusion coefficient maps in early cerebral ischemia. AJNR Am J Neuroradiol 2001;22:1260-7.

20. Loh PS, Butcher KS, Parsons MW, MacGregor L, Desmond PM, Tress BM, et al. Apparent diffusion coefficient thresholds do not predict the response to acute stroke thrombolysis. Stroke 2005;36:2626-31.

21. Kidwell CS, Saver JL, Mattiello J, Starkman S, Vinuela F, Duckwiler G, et al. Thrombolytic reversal of acute human cerebral ischemic injury shown by diffusion/perfusion magnetic resonance imaging. Ann Neurol $2000 ; 47: 462-9$

22. Kidwell CS, Alger JR, Saver JL. Beyond Mismatch: Evolving paradigms in imaging the ischemic penumbra with multimodal magnetic resonance imaging. Stroke 2003;34:2729-35.

23. Röther J, Schellinger PD, Gass A, Siebler M, Villringer A, Fiebach JB, et al. Effect of intravenous thrombolysis on MRI parameters and functional outcome in acute stroke at 6 hours. Stroke 2002;33:2438-45.

24. Neumann-Haefelin T, Wittsack HJ, Wenserski F, Siebler M, Seitz RJ. Diffusion- and perfusion-weighted MRI: The DWI/PWI mismatch region in acute stroke. Stroke 1999;30:1591-7.

25. Kane I, Carpenter T, Chappell F, Rivers C, Armitage P, Sandercock P, et al. Comparison of 10 different magnetic resonance perfusion imaging processing methods in acute ischemic stroke: Effect on lesion size, proportion of patients with diffusion/perfusion mismatch, clinical scores, and radiologic outcomes. Stroke 2007;38:3158-64.

26. Butcher KS, Parsons MW, Davis S, Donnan G. PWI/DWI mismatch: Better definition required. Stroke 2003;34:e215-6.

27. Butcher KS, Parsons M, MacGregor L, Barber PA, Chalk J, Bladin $\mathrm{C}$, et al. Refining the perfusion-diffusion mismatch hypothesis. Stroke 2005;36:1153-9.

28. Heiss WD, Sobesky J. Comparison of PET and DW/PW-MRI in Acute Ischemic Stroke. Keio J Med 2008;57:125-31.

29. Sobesky J, Zaro Weber O, Lehnhardt FG, Hesselmann V, Neveling M, Jacobs A, et al. Does the mismatch match the penumbra? Magnetic resonance imaging and positron emission tomography in early ischemic stroke. Stroke 2005;36:980-5.

30. Warach S. Thrombolysis in stroke beyond three hours: Targeting patients with diffusion and perfusion MRI. Ann Neurol 2002;51:11-3.

31. Schellinger PD, Jansen O, Fiebach JB, Heiland S, Steiner T, Schwab S, et al. Monitoring intravenous recombinant tissue plasminogen activator thrombolysis for acute ischemic stroke with diffusion and perfusion MRI. Stroke 2000;31:1318-28.

32. Parsons MW, Barber PA, Chalk J, Darby DG, Rose S, Desmond PM, et al. Diffusion- and perfusion-weighted MRI response to thrombolysis in stroke. Ann Neurol 2002;51:28-37.

33. Furlan AJ, Eyding D, Albers GW, Al-Rawi Y, Lees KR, Rowley HA, et al.
Dose Escalation of Desmoteplase for Acute Ischemic Stroke (DEDAS): Evidence of safety and efficacy 3 to 9 hours after stroke onset. Stroke 2006:37:1227-31.

34. Hacke W, Albers G, Al-Rawi Y, Bogousslavsky J, Davalos A, Eliasziw M, et al. The Desmoteplase in Acute Ischemic Stroke Trial (DIAS): A phase II MRI-based 9-hour window acute stroke thrombolysis trial with intravenous desmoteplase. Stroke 2005;36:66-73.

35. Marks MP, Olivot JM, Kemp S, Lansberg MG, Bammer R, Wechsler LR, et al. Patients with acute stroke treated with intravenous tPA 3-6 hours after stroke onset: Correlations between MR angiography findings and perfusion- and diffusion-weighted imaging in the DEFUSE study. Radiology 2008;249:614-23.

36. Davis SM, Donnan GA, Parsons MW, Levi C, Butcher KS, Peeters A, et al. Effects of alteplase beyond $3 \mathrm{~h}$ after stroke in the Echoplanar Imaging Thrombolytic Evaluation Trial (EPITHET): A placebocontrolled randomised trial. Lancet Neurol 2008;7:299-309.

37. Christenson S, Parsons M, DE Silva D. Optimal mismatch definitions for detecting treatment response in acute stroke. Cerebrovasc Dis 2008;25:1-192.

38. Dávalos A, Blanco M, Pedraza S, Leira R, Castellanos M, Pumar JM, et al. The clinical-DWI mismatch: A new diagnostic approach to the brain tissue at risk of infarction. Neurology 2004;62:2187-92.

39. Prosser J, Butcher K, Allport L, Parsons M, MacGregor L, Desmond P, et al.Clinical-diffusion mismatch predicts the putative penumbra with high specificity. Stroke 2005;36:1700-4.

40. Barber PA, Hill MD, Eliasziw M, Demchuk AM, Pexman JH, Hudon ME, et al. Imaging of the brain in acute ischaemic stroke: Comparison of computed tomography and magnetic resonance diffusionweighted imaging. J Neurol Neurosurg Psychiatry 2005;76:1528-33.

41. Butcher K, Parsons M, Allport L, Lee SB, Barber PA, Tress B, et al. Rapid assessment of perfusion-diffusion mismatch. Stroke 2008;39:75-81.

42. Lansberg MG, Thijs VN, Bammer R, Olivot JM, Marks MP, Wechsler LR, et al. The MRA-DWI mismatch identifies patients with stroke who are likely to benefit from reperfusion. Stroke 2008;39:2491-6.

43. Singer OC, Humpich MC, Fiehler J, Albers GW, Lansberg MG, Kastrup A, et al. Risk for Symptomatic Intracerebral Hemorrhage after Thrombolysis Assessed by Diffusion-Weighted Magnetic Resonance Imaging. Ann Neurol 2008;63:52-60.

44. Thomalla G, Sobesky J, Köhrmann M, Fiebach JB, Fiehler J, Zaro Weber O, et al. Two tales: Hemorrhagic transformation but not parenchymal hemorrhage after thrombolysis is related to severity and duration of ischemia: MRI study of acute stroke patients treated with intravenous tissue plasminogen activator within 6 hours. Stroke 2007;38:313-8.

45. Tong DC, Adami A, Moseley ME, Marks MP. Relationship between apparent diffusion coefficient and subsequent hemorrhagic transformation following acute ischemic stroke. Stroke 2000;31:2378-84.

46. Selim M, Fink JN, Kumar S, Caplan LR, Horkan C, Chen Y, et al. Predictors of hemorrhagic transformation after intravenous recombinant tissue plasminogen activator: Prognostic value of the initial apparent diffusion coefficient and diffusion-weighted lesion volume. Stroke 2002;33:2047-52.

47. Hayman L, Taber K, Ford J, Bryan R. Mechanisms of MR signal alteration by acute intracerebral blood: Old concepts and new theories. AJNR Am J Neuroradiol 1991;12:899-907.

48. Zamani AA. Imaging of intracranial hemorrhage. In: Rumbaugh CL, Wang A, Tsai FY, editors. Cerebrovascular disease imaging and interventional treatment options. New York: Igaku-Shoin; 1995. p. $232-47$.

49. Linfante I, Llinas RH, Caplan LR, Warach S. MRI features of intracerebral hemorrhage within 2 hours from symptom onset. Stroke 1999;30:2263-7.

50. Butcher KS, Baird T, MacGregor L, Desmond P, Tress B, Davis S. Perihematomal edema in primary intracerebral hemorrhage is plasma derived. Stroke 2004;35:1879-85.

51. Patel MR, Edelman RR, Warach S. Detection of hyperacute primary intraparenchymal hemorrhage by magnetic resonance imaging. Stroke $1996 ; 27: 2321-4$

52. Lin DD, Filippi CG, Steever AB, Zimmerman RD. Detection of intracranial hemorrhage: Comparison between gradient-echo images and 
b(0) images obtained from diffusion-weighted echo-planar sequences. A.JNR Am J Neuroradiol 2001;22:1275-81.

53. Hardy PA, Kucharczyk W, Henkelman RM. Cause of signal loss in MR images of old hemorrhagic lesions. Radiology 1990;174:549-55.

54. Patel R, Edelman R, Warach S. Detection of hyperacute primary intraparenchymal hemorrhge by magnetic resonance imaging. Stroke $1996 ; 27: 2321-24$

55. Kidwell CS, Chalela JA, Saver JL, Starkman S, Hill MD, Demchuk AM, et al. Comparison of MRI and CT for detection of acute intracerebral hemorrhage. JAMA 2004;292:1823-30.

56. Greenberg S, Finklestein S, Schaefer P. Petechial hemorrhages accompanying lobar hemorrhage: Detection by gradient-echo MRI. Neurology 1996;46:1751-54.

57. Fazekas F, Kleinert R, Roob G, Kleinert G, Kapeller P, Schmidt R, Hartung HP. Histopathologic analysis of foci of signal loss on gradient-echo T2*-weighted MR images in patients with spontaneous intracerebral hemorrhage: Evidence of microangiopathy-related microbleeds. A.JNR 1999;20:637-42.

58. Roob G, Lechner A, Schmidt R, Flooh E, Hartung HP, Fazekas F. Frequency and Location of Microbleeds in Patients With Primary Intracerebral Hemorrhage. Stroke 2000;31:2665-9.

59. Roob G, Kleinert R, Seifert T, Lechner A, Kapeller P, Kleinert G, et al. Indications of cerebral micro-hemorrhage in MRI. Comparative histological findings and possible clinical significance. Nervenarzt 1999;70:1082-7.

60. Jeerakathil T, Wolf PA, Beiser A, Hald JK, Au R, Kase CS, et al. Cerebral microbleeds: Prevalence and associations with cardiovascular risk factors in the Framingham Study. Stroke 2004;35:1831-5.

61. Roob G, Schmidt R, Kapeller P, Lechner A, Hartung HP, Fazekas F. MRI evidence of past cerebral microbleeds in a healthy elderly population. Neurology 1999;52:991-4.

62. Chalela JA, Kang DW, Warach S. Multiple cerebral microbleeds: MRI marker of a diffuse hemorrhage-prone state. J Neuroimaging 2004;14:54-7.

63. Kidwell CS, Saver JL, Villablanca JP, Duckwiler G, Fredieu A, Gough K, et al. Magnetic resonance imaging detection of microbleeds before thrombolysis: An emerging application. Stroke 2002;33:95-8.

64. Nighoghossian N, Hermier M, Adeleine P, Blanc-Lasserre K, Derex L, Honnorat $\mathrm{J}$, et al. Old microbleeds are a potential risk factor for cerebra bleeding after ischemic stroke: A gradient-echo T2*-weighted brain MRI study. Stroke 2002;33:735-42.

65. Fiehler J, Albers GW, Boulanger JM, Derex L, Gass A, Hjort N et al. Bleeding Risk Analysis in Stroke Imaging Before ThromboLysis (BRASIL): Pooled analysis of $\mathrm{T} 2 *$-weighted magnetic resonance imaging data from 570 patients. Stroke 2007;38:2738-44.

66. Lee SH, Bae HJ, Kwon SJ, Kim H, Kim YH, Yoon BW, et al. Cerebral microbleeds are regionally associated with intracerebral hemorrhage. Neurology 2004;62:72-6.

67. Ibaraki M, Shimosegawa E, Miura S, Takahashi K, Ito H, Kanno I, et al PET measurements of CBF, OEF, CMRO2 without arterial sampling in hyperacute ischemic stroke: Method and error analysis. Ann Nuc Med 2004;18:35-44.

68. Mahagne MH, Darcourt J, Migneco O, Fournier JP, Thiercelin D, Ducoeur S, et al. Early $(99 \mathrm{~m})$ Tc-ethylcysteinate dimer brain SPECT patterns in the acute phase of stroke as predictors of neurological recovery. Cerebrovasc Dis 2000;10:364-73

69. Alexandrov AV, Grotta JC, Davis SM, Lassen NA. Brain SPECT and thrombolysis in acute ischemic stroke: Time for a clinical trial. J NucIMed 1996;37:1259-62.

70. Giubilei F, Lenzi GL, Di Piero V, Pozzilli C, Pantano P, Bastianello S, et al. Predictive value of brain perfusion single-photon emission computed tomography in acute ischemic stroke. Stroke 1990;21:895-900.

71. Ueda T, Sakaki S, Yuh WTC, Nochide I, Ohta S. Outcome in acute stroke with successful intra-arterial thrombolysis and predictive value of initial single-photon emission-computed tomography. J Cerebr Blood Flow Metab 1999;10:99-108.

Accepted on 09-08-2009

Source of Support: Nil, Conflict of Interest: None declared.

\section{Author Help: Online submission of the manuscripts}

Articles can be submitted online from http://www.journalonweb.com. For online submission, the articles should be prepared in two files (first page file and article file). Images should be submitted separately.

1) First Page File:

Prepare the title page, covering letter, acknowledgement etc. using a word processor program. All information related to your identity should be included here. Use text/rtt/doc/pdf files. Do not zip the files.

2) Article File:

The main text of the article, beginning with the Abstract to References (including tables) should be in this file. Do not include any information (such as acknowledgement, your names in page headers etc.) in this file. Use text/rtf/doc/pdf files. Do not zip the files. Limit the file size to $400 \mathrm{~kb}$. Do not incorporate images in the file. If file size is large, graphs can be submitted separately as images, without their being incorporated in the article file. This will reduce the size of the file.

3) Images:

Submit good quality color images. Each image should be less than 2048 kb (2 MB) in size. The size of the image can be reduced by decreasing the actual height and width of the images (keep up to about 6 inches and up to about 1200 pixels) or by reducing the quality of image. JPEG is the most suitable file format. The image quality should be good enough to judge the scientific value of the image. For the purpose of printing, always retain a good quality, high resolution image. This high resolution image should be sent to the editorial office at the time of sending a revised article.

4) Legends:

Legends for the figures/images should be included at the end of the article file. 